\title{
Genetic variation in the immune system and malaria susceptibility in infants: a nested case-control study in Nanoro, Burkina Faso
}

\author{
Hamatandi Magloire Natama ${ }^{1,2,3^{*}}$, Eduard Rovira-Vallbona' ${ }^{1}$, Meryam Krit ${ }^{1}$, Pieter Guetens ${ }^{1}$, Hermann Sorgho ${ }^{2}$, \\ M. Athanase Somé ${ }^{2}$ Maminata Traoré-Coulibaly², Innocent Valéa ${ }^{2}$, Petra F. Mens ${ }^{4}$, Henk D. F. H. Schallig ${ }^{4}$, \\ Dirk Berkvens ${ }^{1}$, Luc Kestens ${ }^{1,3}$, Halidou Tinto ${ }^{2,5}$ and Anna Rosanas-Urgell ${ }^{1 *}$ (1)
}

\begin{abstract}
Background: Genetic polymorphisms in the human immune system modulate susceptibility to malaria. However, there is a paucity of data on the contribution of immunogenetic variants to malaria susceptibility in infants, who present differential biological features related to the immaturity of their adaptive immune system, the protective effect of maternal antibodies and fetal haemoglobin. This study investigated the association between genetic variation in innate immune response genes and malaria susceptibility during the first year of life in 656 infants from a birth cohort survey performed in Nanoro, Burkina Faso.
\end{abstract}

Methods: Seventeen single nucleotide polymorphisms (SNPs) in 11 genes of the immune system previously associated with different malaria phenotypes were genotyped using TaqMan allelic hybridization assays in a Fluidigm platform. Plasmodium falciparum infection and clinical disease were documented by active and passive case detection. Case-control association analyses for both alleles and genotypes were carried out using univariate and multivariate logistic regression. For cytokines showing significant SNP associations in multivariate analyses, cord blood supernatant concentrations were measured by quantitative suspension array technology (Luminex).

Results: Genetic variants in IL-1 $\beta$ (rs 1 143634) and FCYRIIA/CD32 (rs1801274) —both in allelic, dominant and co-dominant models - were significantly associated with protection from both $P$. falciparum infection and clinical malaria. Furthermore, heterozygote individuals with rs1801274 SNP in FcyRIIA/CD32 showed higher IL-1RA levels compared to wild-type homozygotes $(P=0.024)$, a cytokine whose production is promoted by the binding of IgG immune complexes to $F c y$ receptors on effector immune cells.

Conclusions: These findings indicate that genetic polymorphisms in genes driving innate immune responses are associated to malaria susceptibility during the first year of life, possibly by modulating production of inflammatory mediators.

Keywords: Malaria, Plasmodium falciparum, Immunogenetic variants, Cytokines, Innate immunity

*Correspondence: natamagloire@yahoo.fr; arosanas@itg.be

${ }^{1}$ Department of Biomedical Sciences, Institute of Tropical Medicine, Antwerp, Belgium

Full list of author information is available at the end of the article

\section{Background}

Malaria is a life-threatening infectious disease caused by Plasmodium protozoan parasites and transmitted by Anopheles mosquitoes. Despite global malaria control and elimination efforts, which reduced the number of malaria-related deaths by $50 \%$ since 2000 , malaria

c) The Author(s) 2021. This article is licensed under a Creative Commons Attribution 4.0 International License, which permits use, sharing, adaptation, distribution and reproduction in any medium or format, as long as you give appropriate credit to the original author(s) and the source, provide a link to the Creative Commons licence, and indicate if changes were made. The images or other third party material in this article are included in the article's Creative Commons licence, unless indicated otherwise in a credit line to the material. If material is not included in the article's Creative Commons licence and your intended use is not permitted by statutory regulation or exceeds the permitted use, you will need to obtain permission directly from the copyright holder. To view a copy of this licence, visit http://creativeco mmons.org/licenses/by/4.0/. The Creative Commons Public Domain Dedication waiver (http://creativecommons.org/publicdomain/ zero/1.0/) applies to the data made available in this article, unless otherwise stated in a credit line to the data. 
remains a major public health problem, particularly in pregnant women and children from sub-Saharan Africa $[1,2]$.

Individual risk for malaria infection and disease is complex and multifactorial, and is influenced/modulated by host genetic background [3, 4]. Quantitative genetics have estimated that human genetic factors could explain $25 \%$ of individual variation in susceptibility to clinical malaria in Africa [5]. An example are the numerous studies that have demonstrated a prominent role of red blood cell (RBC) polymorphisms, such as haemoglobininherited disorders (e.g. thalassaemia, sickle cell disease), erythrocyte membrane protein polymorphisms (e.g. ovalocytosis, spherocytosis, Duffy antigen) and erythrocyte enzymatic disorders (e.g. glucose-6-phosphate dehydrogenase) in malaria susceptibility $[6,7]$. On the other hand, there is increasing evidence that identifies polymorphisms in genes related to the immune system as important determinants in susceptibility to malaria infection and disease. Immuno-genetic variants that have been associated with diverse degrees of malaria susceptibility include: (i) polymorphisms in the Human Leucocyte Antigen (HLA), which may affect recognition of parasite antigens [8-10]; (ii) polymorphisms in cytokine related genes, which may affect protein levels and downstream functions, such as production of C-reactive protein and immunoglobulin (Ig) isotype switching [11-16]; (iii) polymorphism in toll-like receptors (TLRs), which may impair the ability of individuals to respond adequately to TLR agonists [17-21]; and (iv) polymorphisms in IgG Fcy receptors, which may affect IgG immune complexes binding and the regulation of the IgG subclass production [22-26].

In Burkina Faso, genetic epidemiology has demonstrated that the wild-type R131 allele (rs1801274) of the FcyRIIA (CD32) and tumour necrosis factor (TNF)-238G allele (rs361525) were associated with protection from clinical malaria and high parasitaemia, respectively, in infants and children until 10 years of age [27-29]. In contrast, in a family based-study, TNF mutations rs3093664 and rs3093662 were associated with increased risk of parasitaemia and clinical malaria [27]. Overall, most of these studies have been conducted in children and adults, whereas the potential effect of immune genetic variants on infants, who are at great risk of malaria [30] and have particular immunologic characteristics (such as an immature adaptive immune system and the potential protective effect of maternal antibodies and fetal haemoglobin [31-35]), has not been investigated.

Previous studies by our group in Burkina Faso described that malaria infections and disease during the first year of life is high and has a marked age and seasonal-dependency [30], that individual heterogeneity in the risk of malaria in this age group is strongly influenced by in utero environment, with a profound effect of past placental malaria on fetal immune system [36]. The study hypothesis was that polymorphisms in genes driving Th1/Th2/innate immune response pathways may also affect the development of fetal innate immunity and thus, contribute to the heterogeneity in malaria susceptibility observed during the first year of life [30, 37, 38]. To address this question, 17 single nucleotide polymorphisms (SNPs) in 11 genes of the innate immune system previously associated with malaria-related phenotypes in African populations (including cytokines, TLRs, Fc $\gamma$ receptors and nitrogen oxide synthase 2, NOS2) [4, 14 , 39-42] were selected to investigated its association with malaria susceptibility in infants living in Nanoro (Burkina Faso), using a nested case-control study design.

\section{Methods}

\section{Study setting}

The study was conducted in the rural health district of Nanoro (Burkina Faso), located in the central-west region at $85 \mathrm{~km}$ from the capital Ouagadougou. Nanoro health district (NHD) has approximately 166,683 inhabitants and comprises 21 peripheral health centres. Malaria transmission is seasonal and hyperendemic with peaks between July and December and overlapping with the rainy season (June to November). Malaria burden in infants is high and strongly dependent on age and season [30]. Between 2014 and 2016, the incidence of clinical malaria was estimated at 1.03 cases per child-year during the first year of life, whereas the age-specific prevalence of asymptomatic infections ranged from $17.7 \%$ at 3 months of age to $31.3 \%$ at 12 months [30].

\section{Study visits and participants}

A birth-cohort study $(\mathrm{n}=734)$ was nested within the COSMIC clinical trial (NCT01941264) to investigate factors that modulate the risk of malaria during the first year of life [30]. COSMIC was a cluster-randomized controlled trial investigating the protective effect of adding community-scheduled screening and treatment of malaria during pregnancy (CSST) to the standard intermittent preventive treatment with sulfadoxine-pyrimethamine (CSST/IPTp-SP, intervention arm) compared to IPTpSP alone (control arm) in Burkina Faso, Benin and The Gambia [43]. Infants from mothers participating into COSMIC trial in Burkina Faso were involved in the birthcohort study. Infants were followed up until 12 months of age following procedures that have been described in detail elsewhere [30, 44]. In brief, malaria infections were detected actively and passively. Active case detection of asymptomatic infections consisted of 4 cross-sectional surveys conducted at 3, 6, 9, and 12 months of age. At 
each survey, blood films and blood spots on filter papers (Whatman 3MM, GE Healthcare Life Sciences) were collected for examination by light microscopy (LM) and quantitative real-time polymerase chain reaction (qPCR), respectively. For passive clinical case detection, mothers were encouraged to bring their offspring to the health centre if they displayed any signs of illness. Infants presenting with fever (axillary temperature $\geq 37.5^{\circ} \mathrm{C}$ ) on examination or history of fever in the previous $24 \mathrm{~h}$ were screened for malaria infection using RDT (SD-Bioline Malaria Ag P.f, Standard Diagnostic, Korea) according to manufacturer's instructions and, if positive, treated according to national guidelines. Malaria diagnosis was subsequently confirmed by LM on Giemsa stained blood films according to standard procedures [45] and qPCR (procedure see below). For the present study, available DNA samples from 694 unrelated singletons were used for SNPs typing.

\section{Detection of $P$. falciparum infections by qPCR}

Parasite and human genomic DNA were extracted from three punches $(5 \mathrm{~mm}$ in diameter) of dried blood spots on filter papers with QIAamp 96 DNA blood kit (Qiagen, Germany) and eluted in $150 \mu \mathrm{L}$ of water, following the manufacturer's recommendations. Five microliters of DNA were used as template for qPCR analysis targeting $P$. falciparum var genes acidic terminal sequence (varATS, $\approx 59$ copies per genome) in StepOne Plus thermocycler (Applied Biosystems), as previously described [46]. The limit of detection of the varATS-qPCR was 0.1 parasite $/ \mu \mathrm{L}$ as described elsewhere [47]. Samples with $\mathrm{Ct}$ value $>39.7$ were considered negative.

\section{SNPs genotyping in fluidigm system}

The SNPs selection for typing was performed based on existing published data. A SNP was included for analysis if: (i) had been previously associated with a malariarelated phenotype (malaria infection, clinical malaria, severe malaria, high parasites density), (ii) had an expected minor allele frequency (MAF) $\geq 5 \%$ in African populations, and (iii) had a functionally tested and/ or validated TaqMan ${ }^{\circledR}$ SNPs genotyping assay. The final SNPs selected for analysis were: TLR1 (rs4833095), TLR4 (rs4986790), TLR9 (rs5743836, rs352139 and rs352140), interleukin (IL)-4 (rs2243250), IL-10 (rs1800890 and rs1800896), IL-17F (rs4715291), IL-1 $\beta$ (rs1143634), TNF (rs1800629 and rs3093664), interferon- $\gamma$ receptor 1 (IFNR1, rs10065633 and rs10213701), nitrogen oxide synthase 2 (NOS2, rs2297518) and FcyRIIA/ CD32 (rs1801274). TaqMan ${ }^{\circledR}$ SNPs genotyping assays were obtained from Applied Biosystems, and genotyping assays performed in GT192.24 dynamic arrays using the BioMark ${ }^{\circledR}$ platform (Fluidigm) at the Department of
Genomics of Common Disease, Imperial College (London, UK). All samples underwent 16 cycles of specific target amplification (STA) in a total reaction volume of $10 \mu \mathrm{L}$. Eight control samples containing known combinations of mutations at selected loci were identified from the Gambian in Western Division (GWD) population based on data from 1000 Genomes Project (http:// phase3browser.1000genomes.org/), and DNA obtained from Coriell Cell Repositories. Both GWD controls and duplicate samples were included in all dynamic arrays. PCR and image processing were carried out on the BioMark ${ }^{\circledR}$ HD080 system (version 4.1.2) to determine SNP genotypes from clusters in each assay. Plots generated by the Fluidigm analysis software were subsequently visually revised to check the cluster profile for each assay and how samples with different genotypes were separated. Allelic calls for all GWD controls were consistent across all runs, and all duplicate samples within each plate had identical allele calls. The genotyping success and the overall call rate was determined per SNP.

\section{Haemoglobin genotyping}

Haemoglobin genotypes were determined by high resolution melting (HRM) analysis, adapting previously described methods [48]. Reactions were conducted in a LightCycler ${ }^{\circledR} 480$ System (Roche) in the presence of LightCycler $^{\circledR} 480$ HRM- Dye (HRM Master 2X, Roche Diagnostics $\mathrm{GmbH}$ ) in a reaction of $20 \mu \mathrm{L}$. LightCycler ${ }^{\circledR}$ 480 Gene Scanning Software (version 1.5) was used to analyse the HRM curves data and determine haemoglobin genotypes. Controls with known haemoglobin genotypes (rs 334 AA [HbAA], rs334 AT [HbAS], rs334 TT [HbSS], rs33930165 GA [HbAC]) were added to every plate.

\section{IL-1 $\beta$ and IL-1RA cytokine levels in cord blood}

Levels of IL-1 $\beta$ and IL-1RA by cord blood cells unstimulated and stimulated with TLR7/8 agonist (a stimulant that demonstrated higher induction of cytokine production than TLR3 and TLR9 agonists in the same cohort) were determined as described previously [36]. Briefly, fresh whole cord blood samples diluted 1:1 with RPMI 1640 (1X, Gibco) were left unstimulated or stimulated with $10 \mu \mathrm{g} / \mathrm{mL}$ of imidazoquinoline (R848, TLR7/8 ligand; InvivoGen, San Diego, USA). Supernatant was collected following incubation for $24 \mathrm{~h}$ at $37{ }^{\circ} \mathrm{C}$ in $5 \%$ $\mathrm{CO}_{2}$, and then frozen at $-80^{\circ} \mathrm{C}$. IL-1 $\beta$ and IL-1RA were quantified with the fluorescent bead-based multiplex immunoassay (Human Cytokine Magnetic 30-Plex Panel kits, Novex ${ }^{\circledR}$, Life Technologies ${ }^{\mathrm{TM}}$, USA) and analysed on a Luminex ${ }^{\circledR} 100 / 200^{\mathrm{TM}}$ instrument using Xponent 3.1 software. 


\section{Definitions and genetic association analysis}

The association between polymorphisms in immune genes and (a) malaria infection or (b) clinical malaria was investigated using case-control analysis. For malaria infection analysis, cases were infants who had at least one $P$. falciparum infection during the first year of life, regardless of symptoms; controls were infants who remained uninfected during the first 12 months of life. Uninfected infants who did not complete the 12-month follow-up were excluded from the analysis.

Clinical malaria cases were infants who experienced at least one clinical episode (presence of $P$. falciparum of any density by qPCR and an axillary temperature $\geq 37.5^{\circ} \mathrm{C}$ or history of fever within the past $24 \mathrm{~h}$ ) from birth to 12 months of age, whereas controls were who did not develop a clinical episode, irrespective of infection status.

Data was analysed using $\mathrm{R}$ statistical package version 3.2.3 [49]. Baseline characteristics of study participants was compared between case and control groups. Continuous data with normal distribution and categorical variables was analysed using Student $t$-test and Pearson's chi-square tests, respectively. Hardy-Weinberg Equilibrium (HWE) was examined using Pearson's chi-square statistical test. SNPs were excluded from the analysis if they had more or equal $10 \%$ of genotype calls missing or a significant genotypic deviation from HWE $(\mathrm{P}<0.001)$. Case-control association analyses for both SNPs alleles and genotypes were assessed using logistic regression in co-dominant, dominant, over-dominant and recessive models [50]. Maternal age, gravidity, infant birth season, sex, low birth weight (LBW), prenatal malaria exposure (defined as maternal peripheral and placental infections and considered as main risk factor regardless of the preventive treatment and SP uptake received during pregnancy [36]), ethnicity and haemoglobin variants were evaluated as potential confounding factors in multivariate analyses. The crude concentrations of IL- $1 \beta$ and IL1RA in un-stimulated and TLR7/8-stimulated samples were $\log$ transformed and compared using analysis of variance (ANOVA) and independent sample $t$-test. Benjamini-Hochberg method was applied to adjust $P$-values for multiple comparisons [51]. A $P$-value $<0.05$ was considered statistically significant.

\section{Results}

\section{Characteristics of cases and controls groups}

Of the 694 infants whose DNA samples were genotyped, $656(94.5 \%)$ completed the 12 months follow-up and were included in the analysis. Overall, 80.3\% (527/656) of infants experienced at least one P. falciparum infection during the first year of life, out of which 78\% (411/527) developed at least one clinical episode. The baseline characteristics of the study participants (cases and controls) are presented in Table 1 . There were no significant differences in maternal characteristics between cases and controls for both malaria infections and clinical episodes. The proportion of infants in different categories of prenatal malaria exposure was significantly different between cases and controls for both clinical cases and malaria infection $(P=0.021$ and $P<0.001$, respectively). In addition, the proportion of infants born during the malaria high-transmission season was significantly higher among clinical malaria controls than cases $(P=0.031)$. In contrast, the proportion of infants born with LBW was significantly higher among clinical malaria cases than controls $(P=0.015)$. There was an ethnic homogeneity among the study participants with most infants (90\%) belonging to the Mossi ethnic group. The proportion of children whose mother received 3 or more SP doses during pregnancy was significantly higher among controls than cases for both malaria infection and clinical episode ( $P=0.006$ and $P=0.034$, respectively). There were no significant differences in haemoglobin genotypes between cases and controls. All the participants were breast feed during the first year of life.

\section{Association between immune genetic variants and malaria infection}

Genotyping success per SNP was $>94 \%$ for all SNPs selected with the exception of TNF rs3093662 SNP (59.7\%), which had $>10 \%$ missing data and was therefore removed from the analysis (Table 2). Call rates for all SNPs were $>96 \%$ for most assays. TLR9 rs3521140 was removed as it deviated from HWE $(P<0.001)$. Among the 15 SNPs included in the final analysis, SNPs rs4833095 (TLR1), rs3093664 (TNF- $\alpha$ ) and rs2297518 (NOS2A) showed a minor allele frequency below $10 \%$ (Table 2).

A first analysis investigated the association between immune genetic variants (allelic and genotypic) and $P$. falciparum infections as detected by qPCR (Table 3 ).

Both univariate and multivariate allele-based analysis showed that infants carrying the mutant allele $\mathrm{A}$ in IL-1 $\beta$ (rs1143634, adjusted odds ratio $(A O R)=0.52$, 95\%CI .34-0.80, $P=0.003$ ) and the mutant allele A in FcyRIIA/CD32 (rs1801274, $\mathrm{AOR}=0.51, \quad 95 \% \mathrm{CI}$ $0.37-0.70, P<0.001$ ) were more likely to be protected against malaria infection than infants with the wild type $\mathrm{G}$ allele. The genotypic analysis (univariate and multivariate) confirmed that polymorphisms in IL-1 $\beta$ (dominant model: AG/AA vs GG, AOR $=0.54,95 \% \mathrm{CI}$ 0.33-0.88, $P=0.014$ ) and iFcyRIIA/CD32 (dominant model: GA/AA vs GG, AOR $=0.44,95 \% \mathrm{CI} 0.28-0.70$, $P<0.001)$ were significantly associated with protection from malaria infection. Furthermore, protection was enhanced among homozygotes individuals in 
Table 1 Characteristics of study participants

\begin{tabular}{|c|c|c|c|c|c|c|}
\hline \multirow[t]{2}{*}{ Variables } & \multicolumn{3}{|c|}{ Malaria infection } & \multicolumn{3}{|l|}{ Clinical malaria } \\
\hline & Cases (527) & Controls (129) & $P$-value & Cases $(\mathrm{N}=411)$ & Controls $(\mathrm{N}=245)$ & $P$-value \\
\hline \multicolumn{7}{|l|}{ Maternal characteristics } \\
\hline Age (years, mean $\pm S D$ ) & $26.5 \pm 6.3$ & $26.2 \pm 6.2$ & 0.641 & $26.4 \pm 6.2$ & $26.4 \pm 6.3$ & 0.988 \\
\hline Gravidity (no. (\%)) & & & 0.176 & & & 0.250 \\
\hline Primigravida & $88(16.7)$ & $25(19.4)$ & & $69(16.8)$ & $44(18.0)$ & \\
\hline Secundigravida & $92(17.5)$ & $14(10.8)$ & & $74(18.0)$ & $32(13.0)$ & \\
\hline Multigravida & $347(65.8)$ & $90(69.8)$ & & $268(65.2)$ & $169(69.0)$ & \\
\hline \multicolumn{7}{|l|}{ MiP preventive strategy } \\
\hline Standard IPTp-SP & $262(49.7)$ & $70(54.3)$ & 0.355 & $212(51.6)$ & $120(49.0)$ & 0.520 \\
\hline CSST/IPTp-SP & $265(50.3)$ & $59(45.7)$ & & $199(48.4)$ & $125(51.0)$ & \\
\hline \multicolumn{7}{|l|}{ SP doses uptake } \\
\hline$<3$ doses & $283(53.7)$ & $52(40.3)$ & 0.006 & $223(54.3)$ & $112(45.7)$ & 0.034 \\
\hline$\geq 3$ doses & $244(46.3)$ & $77(59.7)$ & & $188(45.7)$ & $133(54.3)$ & \\
\hline ITN usage $\mathrm{a}^{\mathrm{a}}$ & $413(78.4)$ & $108(83.7)$ & 0.152 & $326(79.3)$ & $195(79.6)$ & 0.934 \\
\hline \multicolumn{7}{|l|}{ Infant's characteristics } \\
\hline $\begin{array}{l}\text { Birth season [no. in malaria high- } \\
\text { transmission season (\%)] }\end{array}$ & $323(61.3)$ & $84(65.1)$ & 0.422 & $242(58.9)$ & $165(67.4)$ & 0.031 \\
\hline Gender [no. females (\%)] & $272(51.6)$ & $64(49.6)$ & 0.684 & $201(48.9)$ & $135(55.1)$ & 0.125 \\
\hline LBW $(<2500$ g) (no. (\%)) & $45(8.5)$ & $6(4.6)$ & 0.140 & $40(9.7)$ & $11(4.5)$ & 0.015 \\
\hline PME (no. (\%)) & & & $<0.001$ & & & 0.021 \\
\hline Active PM & $113(23.7)$ & $16(13.8)$ & & $87(23.1)$ & $42(19.4)$ & \\
\hline Past PM & $223(46.8)$ & $43(37.0)$ & & $177(47.1)$ & $89(41.0)$ & \\
\hline MPI & $53(11.1)$ & $19(16.4)$ & & $47(12.5)$ & $25(11.5)$ & \\
\hline Non-exposed & $88(18.4)$ & $38(32.8)$ & & $65(17.3)$ & $61(28.1)$ & \\
\hline Ethnicity [no. (\%)] & & & 0.443 & & & 0.410 \\
\hline Mossi & $471(89.4)$ & $122(94.6)$ & & $366(89.1)$ & $227(92.7)$ & \\
\hline Gourounsi & $52(9.9)$ & $6(4.6)$ & & $42(10.2)$ & $16(6.5)$ & \\
\hline Fulani & $3(0.6)$ & $1(0.8)$ & & $2(0.5)$ & $2(0.8)$ & \\
\hline Samo & $1(0.2)$ & $0(0.0)$ & & $1(0.2)$ & $0(0.0)$ & \\
\hline \multicolumn{7}{|l|}{ Haemoglobin ${ }^{b}$} \\
\hline AA & $293(56.8)$ & 75 (58.6) & 0.960 & $235(58.3)$ & $133(55.2)$ & 0.428 \\
\hline$A C$ & $137(26.5)$ & $34(26.6)$ & & $100(24.8)$ & $71(29.5)$ & \\
\hline AS & $67(13.0)$ & $16(12.5)$ & & $53(13.2)$ & $30(12.4)$ & \\
\hline SC & $12(2.3)$ & $2(1.5)$ & & $8(2.0)$ & $6(2.5)$ & \\
\hline SS & $7(1.4)$ & $1(0.8)$ & & $7(1.7)$ & $1(0.4)$ & \\
\hline
\end{tabular}

$P M E$ prenatal malaria exposure, $P M$ Placental malaria, MPI maternal peripheral infection during pregnancy, IPTp-SPIntermittent preventive treatment during pregnancy with sulfadoxine-pyrimethamine, CSST/IPTp-SP community based-scheduled screening and treatment of malaria in addition to the standard IPTp-SP a ITN usage the last night before delivery

b Haemoglobin genotyping was successful for 644 (98.2\%) with a total of 516 infants who experienced P. falciparum infection while 403 developed a clinical case

co-dominant models when comparing the genotypes carrying the mutant allele versus the wild-type genotype for both IL-1 $\beta$ (AA vs GG, AOR $=0.16,95 \% \mathrm{CI}$ $0.04-0.74, P=0.019$ and $\mathrm{AG}$ vs GG, AOR $=0.57,95 \% \mathrm{CI}$ $0.35-0.93, P=0.026)$ and Fc $\gamma$ RIIA/CD32 (AA vs GG, $\mathrm{AOR}=0.28,95 \% \mathrm{CI} 0.14-0.55, P<0.001$ and GA vs GG, $\mathrm{AOR}=0.50,95 \% \mathrm{CI} 0.31-0.81, P=0.004$ ) SNPs (Additional file 1: Table S1). On the contrary, the presence of IL-10 rs1800896 tended to increase the risk of malaria infection in over-dominant model (CT vs TT/CC, $\mathrm{AOR}=1.60,95 \% \mathrm{CI} 0.99-2.61, P=0.057)$.

\section{Association between immune genetic variants and clinical malaria}

The allelic analysis showed that carriage of the mutant allele A in IL-1 $\beta$ rs1143634 SNP (AOR: 0.66, 95\%CI $0.46-0.97 ; P=0.032)$ and mutated allele A in FcyRIIA/ CD32 rs1801274 SNP (AOR: 0.68; 95\%CI 0.52-0.89; 


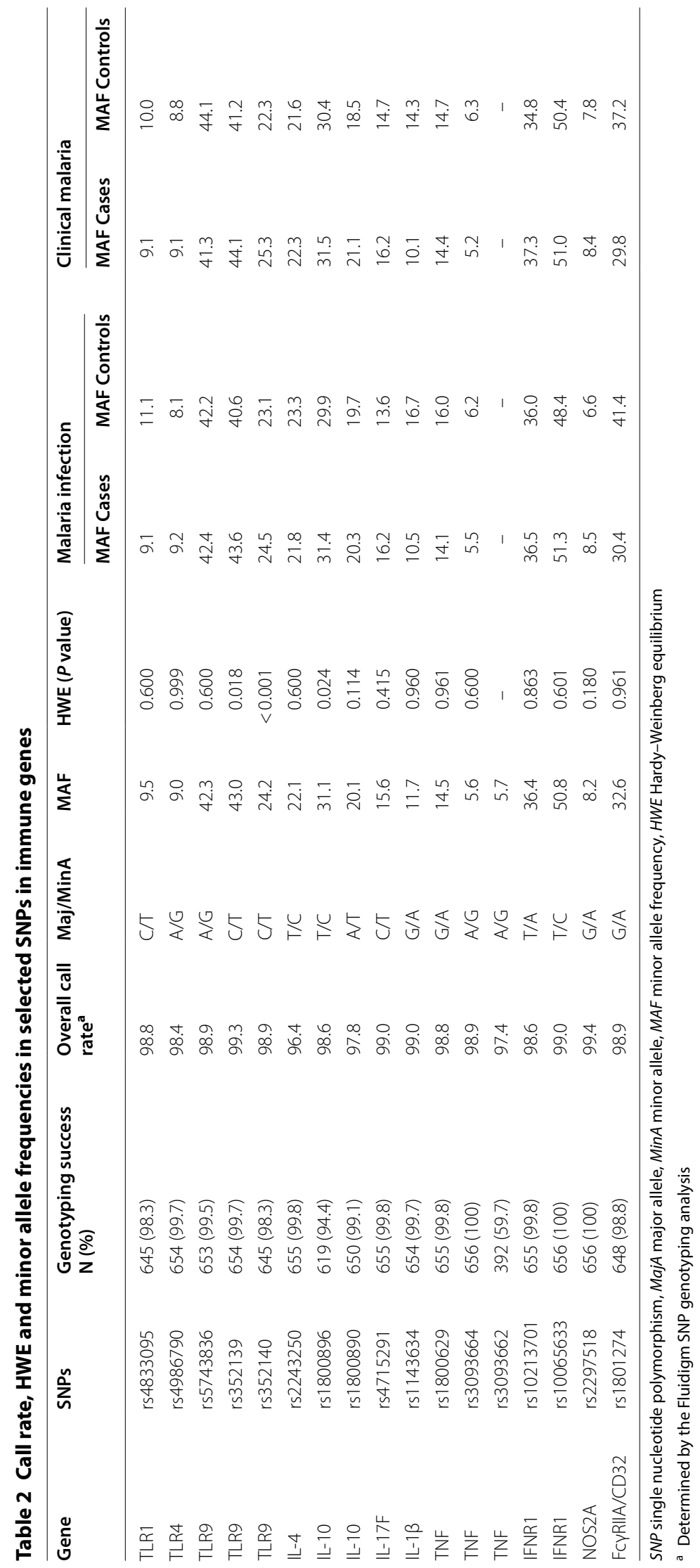


Table 3 SNPs included in the analysis and association with malaria infection

\begin{tabular}{|c|c|c|c|c|c|c|c|}
\hline \multirow[t]{2}{*}{ Gene } & \multirow[t]{2}{*}{ SNPs } & \multirow[t]{2}{*}{ Alleles or genotypes } & \multirow[t]{2}{*}{ Model } & \multicolumn{2}{|c|}{ Univariate analysis } & \multicolumn{2}{|c|}{ Multivariate analysis $^{a}$} \\
\hline & & & & OR $(95 \% \mathrm{Cl})$ & $P$ & AOR $(95 \% C l)$ & $P$ \\
\hline \multicolumn{8}{|c|}{ Allele-based analysis } \\
\hline TLR1 & rs4833095 & T vs $C$ & - & $0.80(0.51-1.24)$ & 0.318 & $0.89(0.54-1.47)$ & 0.660 \\
\hline TLR4 & rs4986790 & Gvs A & - & $1.15(0.70-1.88)$ & 0.581 & $1.44(0.82-2.53)$ & 0.204 \\
\hline TLR9 & rs5743836 & Gvs A & - & $1.01(0.76-1.32)$ & 0.972 & $0.98(0.72-1.33)$ & 0.760 \\
\hline TLR9 & rs352139 & Tvs $C$ & - & $1.13(0.85-1.49)$ & 0.384 & $1.22(0.89-1.66)$ & 0.219 \\
\hline IL-4 & rs 2243250 & C vs T & - & $0.91(0.66-1.27)$ & 0.606 & $0.89(0.63-1.27)$ & 0.534 \\
\hline IL-10 & rs1800896 & C vs T & - & $1.07(0.78-1.46)$ & 0.664 & $1.07(0.76-1.53)$ & 0.685 \\
\hline IL-10 & rs1800890 & Tvs A & - & $1.04(0.73-1.46)$ & 0.835 & $1.10(0.75-1.61)$ & 0.614 \\
\hline IL-17F & rs4715291 & Tvs $C$ & - & $1.23(0.83-1.82)$ & 0.305 & $1.11(0.72-1.71)$ & 0.629 \\
\hline $\mathbb{L L}-1 \beta$ & rs 1143634 & $A \vee s G$ & - & $0.58(0.40-0.86)$ & 0.006 & $0.52(0.34-0.80)$ & 0.003 \\
\hline TNF-a & rs1800629 & $A \vee s G$ & - & $0.86(0.59-1.26)$ & 0.444 & $0.91(0.60-1.38)$ & 0.669 \\
\hline TNF-a & rs3093664 & Gvs A & - & $0.88(0.50-1.56)$ & 0.663 & $0.93(0.48-1.78)$ & 0.826 \\
\hline IFNR1 & rs10213701 & $A v s T$ & - & $1.02(0.77-1.35)$ & 0.891 & $0.97(0.71-1.34)$ & 0.888 \\
\hline IFNR1 & rs10065633 & C vs T & - & $1.12(0.84-1.47)$ & 0.407 & $1.20(0.88-1.63)$ & 0.238 \\
\hline NOS2A & rs2297518 & $A \vee s G$ & - & $1.32(0.77-2.26)$ & 0.306 & $1.28(0.71-2.33)$ & 0.408 \\
\hline FCYRIIA/CD32 & rs1801274 & $A \vee s G$ & - & $0.62(0.47-0.82)$ & $<0.001$ & $0.51(0.37-0.70)$ & $<0.001$ \\
\hline \multicolumn{8}{|c|}{ Genotype-based analysis $^{b}$} \\
\hline TLR1 & rs4833095 & TT vs TC/CC & Recessive & $0.60(0.11-3.14)$ & 0.423 & $0.57(0.10-3.18)$ & 0.521 \\
\hline TLR4 & rs4986790 & GA/GG vs $A A$ & Dominant & $1.11(0.66-1.86)$ & 0.828 & $1.43(0.79-2.58)$ & 0.237 \\
\hline TLR9 & rs5743836 & GG vs GA/AA & Recessive & $0.87(0.54-1.40)$ & 0.565 & $0.95(0.56-1.62)$ & 0.861 \\
\hline TLR9 & rs352139 & TT vs TC/CC & Recessive & $1.30(0.79-2.14)$ & 0.291 & $1.57(0.89-2.77)$ & 0.117 \\
\hline$\| \mathrm{L}-4$ & rs 2243250 & TC vs TT/CC & Over-dominant & $0.93(0.62-1.40)$ & 0.730 & $0.91(0.58-1.43)$ & 0.695 \\
\hline IL-10 & rs1800896 & CT vs TT/CC & Over-dominant & $1.32(0.86-2.02)$ & 0.201 & $1.60(0.99-2.61)$ & 0.057 \\
\hline IL-10 & rs1800890 & TA vs TT/AA & Over-dominant & $1.33(0.86-2.07)$ & 0.203 & $1.52(0.93-2.48)$ & 0.098 \\
\hline IL-17F & rs4715291 & TT vs TC/CC & Recessive & $1.15(0.73-1.83)$ & 0.537 & $1.28(0.35-4.61)$ & 0.710 \\
\hline $\mathbb{L L}-1 \beta$ & rs1143634 & $A G / A A$ vs $G G$ & Dominant & $0.58(0.37-0.89)$ & 0.013 & $0.52(0.32-0.84)$ & 0.007 \\
\hline TNF-a & rs1800629 & AA vs GA/GG & Recessive & $1.34(0.29-6.13)$ & 0.704 & $1.41(0.28-7.10)$ & 0.677 \\
\hline TNF-a & rs3093664 & GA vs GG/AA & Over-dominant & $0.74(0.41-1.35)$ & 0.325 & $0.77(0.39-1.52)$ & 0.454 \\
\hline IFNR1 & rs10065633 & TC vs CC/TT & Over-dominant & $0.89(0.61-1.32)$ & 0.574 & $0.90(0.58-1.37)$ & 0.619 \\
\hline IFNR1 & rs10213701 & TA vs AA/TT & Over-dominant & $0.85(0.58-1.25)$ & 0.415 & $0.86(0.56-1.32)$ & 0.492 \\
\hline NOS2A & rs2297518 & GA/AA vs GG & Over-dominant & $1.32(0.74-2.34)$ & 0.342 & $1.30(0.69-2.46)$ & 0.421 \\
\hline FCyRIIA/CD32 & rs1801274 & GAVAA vs GG & Dominant & $0.52(0.34-0.78)$ & 0.001 & $0.45(0.28-0.70)$ & $<0.001$ \\
\hline
\end{tabular}

Significant $P$ values $<0.05$ are indicated in italics

a Adjusted by mother's age, gravidity, Birth season, baby's sex, LBW, Prenatal malaria exposure, ethnicity, Haemoglobin variants

b Genotype-based analysis for dominant, over-dominant and recessive models. Models that showed lowest $P$ values are shown

$P=0.005)$ were associated with protection from clinical malaria. The same associations were observed in the genotype-based analysis showing that individuals with AA genotype in IL-1 $\beta$ rs1143634 SNP (recessive model: AA vs AG/GG, AOR $=0.14,95 \%$ CI 0.03-0.78, $P=0.024)$ and those with GA/AA genotypes in FcyRIIA/ CD32 rs1801274 SNP (dominant model: GA/AA vs GG, $\mathrm{AOR}=0.58,95 \% \mathrm{CI} 0.41-0.84, P=0.003)$ were more likely to be protected against clinical malaria (Table 4). In the co-dominant model, the risk of clinical malaria was only reduced among homozygote AA carriers of
IL-1 $\beta$ rs1143634 SNP (AA vs GG, AOR $=0.14,95 \% \mathrm{CI}$ $0.03-0.75, P=0.022$ ), while both AA mutant homozygote and GA heterozygote individuals for FcyRIIA/ CD32 rs1801274 SNP were significantly protected (AA vs $\mathrm{GG}, \mathrm{AOR}=0.48,95 \% \mathrm{CI} 0.26-0.87, P=0.017$ and GA vs $\mathrm{GG}, \mathrm{AOR}=0.61,95 \% \mathrm{CI} 0.42-0.89, P=0.010$ ) (Additional file 2: Table S2). No significant association was found between the selected SNPs with increased risk of clinical malaria during the first year of life. However, a marginal increase risk was observed among infants carrying the TA genotype for rs1800890 SNP in IL-10 locus 
Table 4 SNPs included in the analysis and association with clinical malaria

\begin{tabular}{|c|c|c|c|c|c|c|c|}
\hline \multirow[t]{2}{*}{ Gene } & \multirow[t]{2}{*}{ SNPs } & \multirow[t]{2}{*}{ Alleles/genotypes } & \multirow[t]{2}{*}{ Model } & \multicolumn{2}{|c|}{ Univariate analysis } & \multicolumn{2}{|c|}{ Multivariate analysis $^{a}$} \\
\hline & & & & OR $(95 \% \mathrm{Cl})$ & $P$ & OR $(95 \% \mathrm{Cl})$ & $P$ \\
\hline \multicolumn{8}{|c|}{ Allele-based analysis } \\
\hline TLR1 & rs4833095 & Tvs $C$ & - & $0.90(0.62-1.33)$ & 0.608 & $0.96(0.63-1.46)$ & 0.844 \\
\hline TLR4 & rs4986790 & Gvs A & - & $1.04(0.70-1.54)$ & 0.838 & $1.19(0.76-1.87)$ & 0.442 \\
\hline TLR9 & rs5743836 & Gvs A & - & $0.89(0.71-1.12)$ & 0.324 & $0.82(0.64-1.06)$ & 0.137 \\
\hline TLR9 & rs352139 & Tvs $C$ & - & $1.13(0.90-1.42)$ & 0.296 & $1.09(0.84-1.40)$ & 0.524 \\
\hline IL-4 & rs 2243250 & C vs T & - & $1.04(0.79-1.36)$ & 0.772 & $0.97(0.72-1.31)$ & 0.857 \\
\hline $\mid \mathrm{LL}-10$ & rs1800896 & C vs T & - & $1.05(0.82-1.35)$ & 0.676 & $1.00(0.76-1.33)$ & 0.982 \\
\hline IL-10 & rs1800890 & Tvs A & - & $1.17(0.88-1.56)$ & 0.268 & $1.14(0.83-1.56)$ & 0.423 \\
\hline IL-17F & rs4715291 & Tvs $C$ & - & $1.12(0.82-1.53)$ & 0.462 & $0.98(0.70-9.07)$ & 0.918 \\
\hline $\mathbb{L L}-1 \beta$ & rs1143634 & $A \vee s G$ & - & $0.68(0.48-0.95)$ & 0.025 & $0.66(0.46-0.97)$ & 0.032 \\
\hline TNF- $a$ & rs1800629 & $A$ vs $G$ & - & $0.97(0.70-1.33)$ & 0.843 & $1.08(0.76-1.53)$ & 0.682 \\
\hline TNF-a & rs3093664 & Gvs A & - & $0.82(0.51-1.32)$ & 0.406 & $0.73(0.43-1.25)$ & 0.259 \\
\hline IFNR1 & rs10213701 & $A \vee s T$ & - & $1.11(0.88-1.41)$ & 0.361 & $1.15(0.89-1.50)$ & 0.283 \\
\hline IFNR1 & rs10065633 & C vsT & - & $1.02(0.82-0.82)$ & 0.843 & $1.06(0.82-1.36)$ & 0.644 \\
\hline NOS2A & rs2297518 & $A$ vs $G$ & - & $1.09(0.72-1.65)$ & 0.682 & $1.32(0.83-2.10)$ & 0.239 \\
\hline FCYRIIA/CD32 & rs1801274 & $A \vee s G$ & - & $0.71(0.56-0.90)$ & 0.005 & $0.68(0.52-0.89)$ & 0.005 \\
\hline \multicolumn{8}{|c|}{ Genotype-based analysis ${ }^{b}$} \\
\hline TLR1 & rs4833095 & TT vs TC/CC & Recessive & $0.44(0.10-1.99)$ & 0.286 & $0.50(0.11-2.36)$ & 0.388 \\
\hline TLR4 & rs4986790 & GA/GG vs AA & Dominant & $1.02(0.67-1.56)$ & 0.910 & $1.28(0.79-2.06)$ & 0.313 \\
\hline TLR9 & rs5743836 & GG vs GA/AA & Recessive & $0.81(0.54-1.20)$ & 0.295 & $0.77(0.50-1.20)$ & 0.250 \\
\hline TLR9 & rs352139 & TT vs TC/CC & Recessive & $1.33(0.90-1.98)$ & 0.155 & $1.33(0.86-2.06)$ & 0.197 \\
\hline$\| \mathrm{L}-4$ & rs 2243250 & TC vs TT/CC & Over-dominant & $0.96(0.68-1.35)$ & 0.818 & $0.83(0.57-1.20)$ & 0.326 \\
\hline IL-10 & rs1800896 & CT vs TT/CC & Over-dominant & $1.07(0.76-1.50)$ & 0.690 & $1.29(0.88-1.88)$ & 0.185 \\
\hline$\| \mathrm{L}-10$ & rs1800890 & TA vs TT/AA & Over-dominant & $1.41(0.99-2.01)$ & 0.059 & $1.48(0.99-2.19)$ & 0.052 \\
\hline IL-17F & rs4715291 & TT vs TC/CC & Recessive & $1.29(0.52-3.02)$ & 0.583 & $0.92(0.35-2.46)$ & 0.882 \\
\hline$I L-1 \beta$ & rs1143634 & $A A v s A G / G G$ & Recessive & $0.29(0.07-1.18)$ & 0.086 & $0.14(0.03-0.78)$ & 0.024 \\
\hline TNF-a & rs1800629 & AA vs GA/GG & Recessive & $1.34(0.41-4.41)$ & 0.626 & $2.15(0.54-8.59)$ & 0.279 \\
\hline TNF-a & rs3093664 & GA vs GG/AA & Over-dominant & $0.85(0.50-1.42)$ & 0.529 & $0.70(0.40-1.25)$ & 0.230 \\
\hline IFNR1 & rs10065633 & TC vs CC/TT & Over-dominant & $1.03(0.75-1.41)$ & 0.869 & $1.14(0.80-1.61)$ & 0.476 \\
\hline IFNR1 & rs10213701 & TA/AA vs TT & Dominant & $1.13(0.82-1.55)$ & 0.468 & $1.20(0.84-1.70)$ & 0.331 \\
\hline NOS2A & rs2297518 & GA/AA vs GG & Dominant & $1.11(0.71-1.73)$ & 0.656 & $1.30(0.79-2.14)$ & 0.307 \\
\hline FCYRIIA/CD32 & rs1801274 & GA/AA vs GG & Dominant & $0.65(0.47-0.89)$ & 0.008 & $0.58(0.41-0.84)$ & 0.003 \\
\hline
\end{tabular}

Significant $P$ values $<0.05$ are indicated in italics

a Adjusted by mother's age, gravidity, birth season, baby's gender, low birth weight, Prenatal malaria exposure, ethnicity, Haemoglobin variant

${ }^{\text {b }}$ Genotype-based analysis for dominant, over-dominant and recessive models. Models that showed lowest $P$ values $(P)$ are shown

(over-dominant model: $\mathrm{TA}$ vs $\mathrm{TT} / \mathrm{AA}, \mathrm{AOR}=1.48$, 95\%CI 0.99-2.19, $P=0.052$ ) (Table 4).

\section{Association between genetic polymorphisms and cytokine levels in cord blood}

Next it was assessed whether polymorphisms in IL-1 $\beta$ (rs1143634) were associated with immune effector phenotypes. Therefore, IL-1 $\beta$ levels were quantified in a sub-group of infants) for whom un-stimulated and
TLR7/8-stimulated cord blood samples were available $(\mathrm{N}=313)$. Figure 1 shows that IL- $1 \beta$ levels did not differ across rs1143634 genotypic groups for both unstimulated and TLR-7/8-stimulated samples $(P=0.682$ and $P=0.707$, respectively).

The association between FcyRIIA/CD32 rs1801274 SNP and IL-1RA levels was also investigated, since binding of FcyRIIA/CD32 to immune complexes induces IL-1RA production [52, 53]). In this case, significant differences in IL-1RA levels across FcrRIIA/CD32 


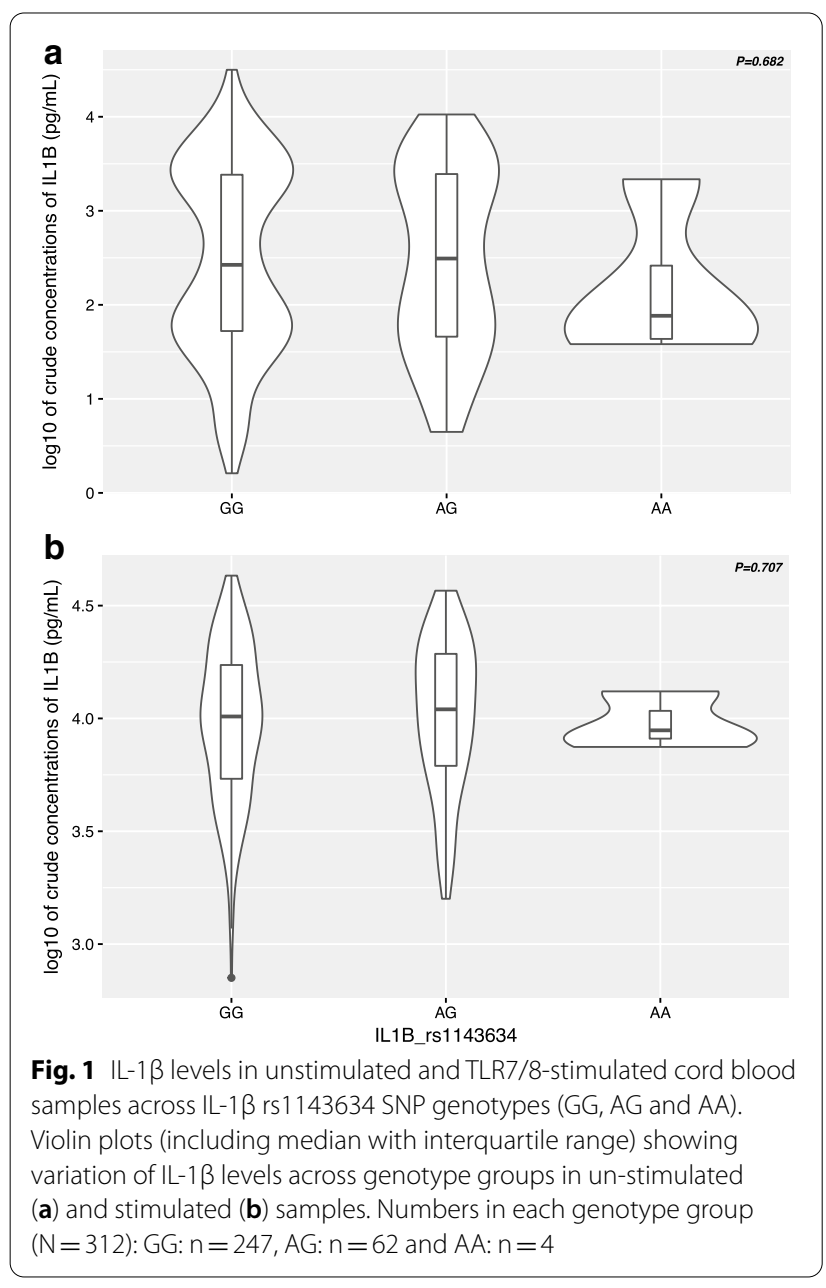

rs1801274 genotypes upon TLR7/8 stimulation of cord blood samples were observed ( $P=0.016$, Fig. $2 b)$. Individuals with GA genotype had higher levels of IL-1RA compared with that of infants carrying the wild-type GG genotype $(P=0.024)$, while no significant difference was observed for individuals carrying the AA genotype $(P=0.487)$. Differences of IL-1RA levels between genotypes in un-stimulated cord blood samples was not significant $(P=0.619$, Fig. 2a).

\section{Discussion}

This study investigated associations between genetic variants in immune genes and malaria susceptibility in infants in Burkina Faso. The study focused on cytokine, TLR, FcyRIIA/CD32 and NOS2A genes given their key role in innate immune responses against pathogens in early infancy. SNPs in FcyRIIA/CD32 (rs1801274) and in IL-1 $\beta$ (rs1143634) were significantly associated with protection from both malaria infection and clinical disease during the first year of life and FcyRIIA/CD32 SNP rs1801274 genotypes were associated with IL-1RA levels,
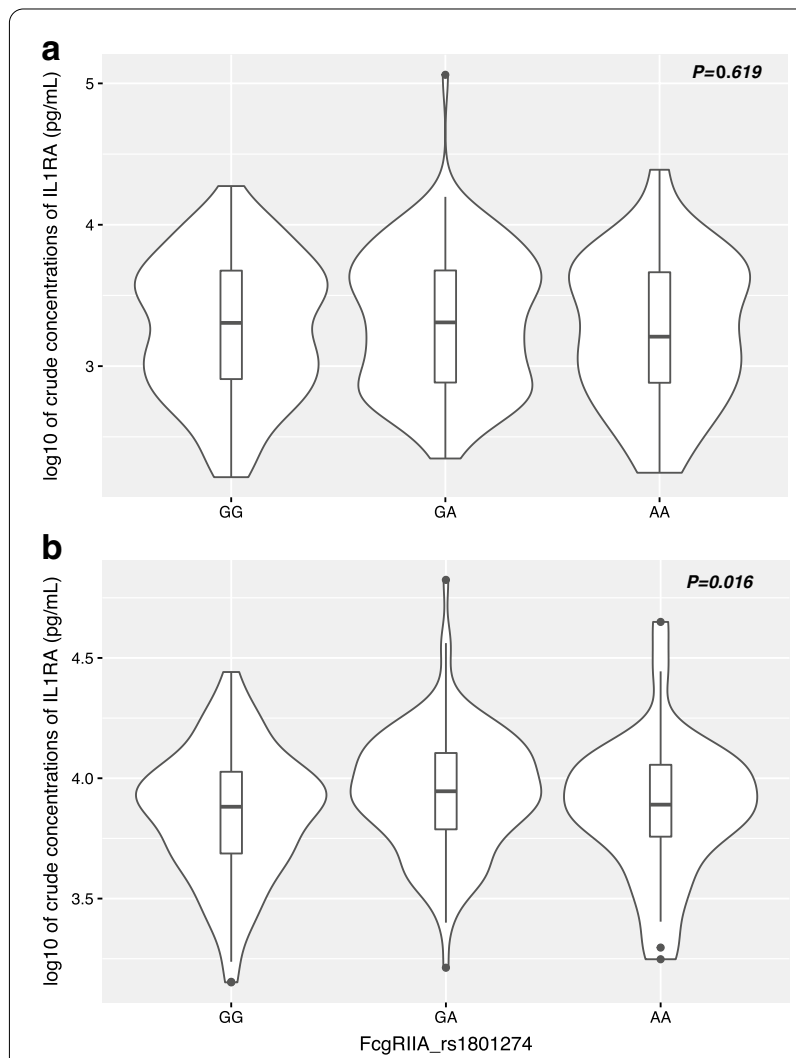

Fig. 2 IL-1RA levels in unstimulated and TLR7/8-stimulated cord blood samples across FcyRIIA/CD32 gene (rs1801274) genotypes (GG, AG and AA). Violin plots (including median with interquartile range) showing variation of IL-1RA levels across genotype groups in un-stimulated (a) and stimulated (b) samples. Numbers in each genotype group ( $N=309)$ : GG: $n=145, G A: n=128$ and $A A: n=36$

a cytokine involved in inflammatory responses to malaria infection.

A number of studies have explored the association between FcyRIIA/CD32 rs1801274 SNP and malaria susceptibility resulting in contradictory findings $[28,29,40$, $54-59]$. However, only a limited number included infants and the majority of those focused on severe malaria (e.g. severe malarial anaemia and high-density parasitaemia) $[25,60]$. The strongest evidence of FcyRIIA/CD32 rs1801274 (A allele; variant $\mathrm{H}$ ) protection against bloodstage malaria infections comes from a meta-analysis study on 6928 subjects from Africa and Asia [40]. Results in this study show a similar protective effect of the A allele of the FcyRIIA/CD32 rs1801274 SNP against both malaria infection and disease, with associations confirmed in both co-dominant (AA $v s$ GG and GA $v s$ GG) and dominant (GA/AA vs GG) models after adjusting by potential confounding factors.

Fc $\gamma$ receptors (Fc $\gamma$ RI, Fc $\gamma$ RII, Fc $\gamma$ RIII) bind to the Fc domain of IgG on immune cells and thereby mediate the initiation of a variety of immunological responses 
including antigen presentation, phagocytosis, cytotoxicity, release of inflammatory mediators and the modulation of immune responses [61, 62]. Therefore, Fc $\gamma$ Rs are an important link between cellular and humoral immunity in host defence against malaria infection [62]. FcyRIIA, which is expressed on the surface of all types of cells of the immune system, is a low affinity receptor for monomeric IgG, but binds IgG immune complexes efficiently [63]. Fc $\gamma$ RIIA/CD32 rs1801274 has been found to alter the gene function in vitro, by extending the preferential affinity of the wild-type $G$ allele for IgG1 and IgG3 towards affinity for IgG2 [57, 64]. Therefore, it's reasonable to believe that the efficient binding to IgG2 while retaining its affinity to IgG1 and IgG3 increase the antibody mediated protective effect against malaria. It has been also suggested that differential binding between immune complexes and Fcy receptors due to polymorphisms in FcyRIIA induce changes in the ability of immune cells to respond to $P$. falciparum infection through production of inflammatory mediators [58, 62, 64]. Here, the effect of rs1801274 SNP in FcyRIIA/CD32 gene on unstimulated and TLR-stimulated IL-1RA production in cord blood samples was tested, as it has been previously demonstrated that FcyRIIA/CD32 plays a key role in IL-1RA production through binding of immune complexes to monocyte, macrophage and neutrophil lineage cells $[52,53]$. Several studies have demonstrated the clinical relevance of IL-1RA in immune responses to malaria [65-68], which acts modulating disease severity by competing with the pro-inflammatory cytokines IL- $1 \alpha$ and IL- $1 \beta$ for binding sites on the IL- 1 type I receptor and inhibits IL-1 signalling [69]. While there were no significant differences in the spontaneous production of IL-1RA by cord blood cells across FcyRIIA/ CD32 rs1801274 genotypes, upon TLR7/8 stimulation, individuals with GA genotype had significantly higher levels of IL-1RA compared with those with the wild type GG genotype. Remarkably, in infants from this cohort TLR7/8-induced IL-1RA production in cord blood was an independent predictor of malaria protection during the first year of life [36]. Therefore, study findings support the hypothesis that variability in susceptibility to malaria across FcyRIIA/CD32 rs1801274 genotypes is mediated, at least partially, by changes in immune complex-mediated cytokine production. In addition to the protective effect of IgG1 and IgG3, FcgR2A mutant carriers are likely to be protected due to the high affinity of the A allele product to IgG2 possibly by improving the antibody mediated protective effect. Given that this protective effect could be effective through the mediation of a variety of immunological responses including inflammatory mediators, level of IL1-RA could represent one of the changes in innate immune response to malaria infection between the mutant $\mathrm{A}$ and the wild-type $\mathrm{G}$ allele carriers. The fact that a significant difference was not observed for homozygous allele could be due to limited number of individuals carrying the AA genotype in the subset of data analysed.

The second genetic variant in immune genes associated with malaria protection in this study was the IL-1 $\beta$ rs1143634 SNP. The carriage of IL-1 $\beta$ rs1143634 mutated allele A (both in homozygosity and heterozygosity) was associated with a decreased risk of malaria infection ( $84 \%$ and $43 \%$ reduction, respectively). In addition, AA homozygote carriers (but not AG) had a significantly reduced risk of clinical malaria, suggesting an enhanced protective effect of AA homozygosity. This is the first study to report a protective effect of IL-1 $\beta$ rs1143634 SNP against malaria infection and uncomplicated disease in infants. Previous studies assessing associations of IL-1 $\beta$ rs1143634 and malaria susceptibility reported heterogeneous findings in children including an association with higher peripheral parasitaemia in Ghanaian children $(\mathrm{n}=461$; aged $1-12$ years $)$ [70], an association with severe malaria in Gambian children $(\mathrm{n}=1420$; aged $<5$ years) [71], or no significant association with malaria susceptibility in Cameroonian children ( $n=1862$; aged $1-14$ years) [72]. These contrasting results may be attributed to differences in age groups, ethnicity and/or malaria phenotype evaluated.

IL- $1 \beta$ is a pro-inflammatory cytokine that is implicated in the first line of defence against pathogens, including the hepatic and erythrocytic stages of malaria parasites [73-75]. However, high levels of sustained IL-1 $\beta$ production may induce pathogenic effects that promote disease manifestation and severity [76-78]. Previous studies have already demonstrated a correlation between IL-1 $\beta$ rs1143634 SNP and increased in vitro production of IL-1 $\beta[79,80]$. To investigate whether the observed protective effect of the rs1143634 SNP in this study was related to functional changes in IL- $1 \beta$ production, IL- $1 \beta$ levels in cord blood were compared across genotypic groups but no significant differences were observed neither in un-stimulated or TLR7/8-stimulated samples. Genetic variations occurring in genes encoding inflammatory cytokines can have a direct effect on the innate immune responses to malaria infection and disease manifestation. In this regard, the protective effect of the IL- $1 \beta$ rs 1143634 could be due to the modulation of IL- $1 \beta$ level towards a protective effect instead to levels that could be harmful.

However, these findings should be interpreted with caution due to very low number of individuals carrying the AA genotype among the subset of infants that could 
be analysed $(\mathrm{n}=4)$ and, therefore, potential functional changes in IL- $1 \beta$ production by IL- $1 \beta$ rs1143634 SNP cannot be ruled out. Likewise, it is worth noting that IL- $1 \beta$ production is likely to be influenced by other SNPs in IL-1 $\beta$ gene, as well as polymorphisms in inflammatory mediators such as TNF [81-84] that were not assessed in this study. Correlation between SNP of interest and cytokine levels in different genotypes should be further explored in different malaria phenotypes (severe, uncomplicated, infection) in the field to elucidate these contrasting results.

Other genes coding for major TLRs (i.e. TLR1, 4 and 9) and cytokines (i.e. IL-4, IL-10, IL-17F and TNF) did not show significant association with malaria infection and clinical episodes, although rs1800890 SNPs in IL-10 gene showed a trend towards increased susceptibility to malaria infection or clinical episode in multivariate analysis $(P=0.057$ and $P=0.052$, respectively). IL-10 is a key immuno-regulator of immunity to infections [85] and has been associated with various malaria-related phenotypes, such as uncomplicated malaria, severe malaria, severe malaria anaemia and high parasite density [86-91]. In addition, a number of studies have reported associations between IL-10 SNPs and susceptibility to malaria with either protective effects in some SNPs and increased susceptibility in others [72, 92-95]. Therefore, this tendency of an increased risk of malaria among carriers of rs 1800890 SNP in IL-10 gene, could results from changes that do not allow adequate modulation of inflammatory responses since IL-10 is an anti-inflammatory cytokine.

\section{Conclusion}

This study showed that the mutated A allele of IL- $1 \beta$ rs1143634 and mutated A allele of FcyRIIA/CD32 rs1801274 are associated with protection against both malaria infections and uncomplicated disease during the first year of life. These findings suggest that genetic polymorphisms in genes driving innate immune responses could condition malaria susceptibility during the first year of life, possibly by modulating production of inflammatory mediators. Future investigations on the epistatic effects of immune genes polymorphisms described here and functional studies may contribute to further understand how host genetic variation influences malaria susceptibility in infancy.

\section{Supplementary Information}

The online version contains supplementary material available at https://doi. org/10.1186/s12936-021-03628-y.

Additional file 1: Table S1. Genotypic-based association analysis for malaria infection using co-dominant models.
Additional file 2: Table S2. Genotypic-based association analysis for clinical malaria using co-dominant models.

\section{Acknowledgements}

We sincerely thank mothers and their offspring for participating into this study. We acknowledge the research teams at the Clinical Research Unit of Nanoro (CRUN) in Burkina Faso and at the Malariology Unit, Institute of Tropical Medicine (ITM, Belgium) for this successful collaborative research project.

\section{Authors' contributions}

HMN, ERV and ARU conceived and designed the study. HMN, HS, MAS, MTC, SS, SHZ, OS, SCZ and IV supervised data and samples collection in the field. HMN and ERV designed and interpreted the genetic analysis. HMN, ERV and ARU analysed the data. IV, PFM, HDFHS, HT, LK and ARU contributed for reagents/materials/analysis tools. HMN, ERV and ARU were the major contributors in drafting the manuscript. All authors read and approved the final manuscript.

\section{Funding}

This work was supported by the Belgium Directorate General for Development Cooperation (DGD, Grant number FA3-III ITM-CRUN) under the Framework Agreement Program between ITM and CRUN (2014-2016) and COSMIC project funded by European Union Seventh Framework Programme (FP7/2002-2016) under grant agreement no 305662-COSMIC.

\section{Availability of data and materials}

The datasets during and/or analysed during the current study available from the corresponding author on reasonable request.

\section{Ethics approval and consent to participate}

Written informed consent was obtained from mothers during antenatal care visits prior to delivery. The study received ethical approvals from the institutional ethics committees of Centre Muraz, Bobo Dioulasso, Burkina Faso (0062014/CE-CM), the Institute of Tropical Medicine, Antwerp, Belgium (953/14), and University Hospital in Antwerp, Belgium (14/26/277).

\section{Competing interests}

The authors declare that they have no competing interests.

\section{Author details}

${ }_{1}$ Department of Biomedical Sciences, Institute of Tropical Medicine, Antwerp, Belgium. ${ }^{2}$ Unité de Recherche Clinique de Nanoro, Institut de Recherche en Sciences de La Santé, Nanoro, Burkina Faso. ${ }^{3}$ Department of Biomedical Sciences, University of Antwerp, Antwerp, Belgium. ${ }^{4}$ Department of Medical Microbiology-Parasitology Unit, Academic Medical Centre, Amsterdam University Medical Centres, Amsterdam, The Netherlands. ${ }^{5}$ Centre Muraz, Bobo Dioulasso, Burkina Faso.

Received: 30 March 2020 Revised: 3 February 2021 Accepted: 5 February 2021

Published online: 16 February 2021

\section{References}

1. WHO. World malaria report 2016. Geneva: World Health Organization; 2016.

2. WHO. World malaria report 2017. Geneva: World Health Organization; 2017.

3. Hill AV. Genetics of infectious disease resistance. Curr Opin Genet Dev. 1996;6:348-53.

4. de Mendonça VRR, Goncalves MS, Barral-Netto M. The host genetic diversity in malaria infection. J Trop Med. 2012;2012:940616.

5. Mackinnon MJ, Mwangi TW, Snow RW, Marsh K, Williams TN. Heritability of malaria in Africa. PLoS Med. 2005;2:1253-9.

6. Driss A, Hibbert JM, Wilson NO, lqbal SA, Adamkiewicz TV, Stiles JK. Genetic polymorphisms linked to susceptibility to malaria. Malar J. 2011;10:271. 
7. Marquet S. Overview of human genetic susceptibility to malaria: From parasitemia control to severe disease. Infect Genet Evol. 2018;66:399-409.

8. Hill AV, Allsopp CE, Kwiatkowski D, Anstey NM, Twumasi P, Rowe PA, et al. Common West African HLA antigens are associated with protection from severe malaria. Nature. 1991;352:595-600.

9. May J, Lell B, Luty AJ, Meyer CG, Kremsner PG. HLA-DQB1*0501-restricted Th1 type immune responses to Plasmodium falciparum liver stage antigen 1 protect against malaria anemia and reinfections. J Infect Dis. 2001;183:168-72.

10. Flori L. Linkage of mild malaria to the major histocompatibility complex in families living in Burkina Faso. Hum Mol Genet. 2003;15(12):375-8.

11. Zhang G, Manaca MN, McNamara-Smith M, Mayor A, Nhabomba A, Berthoud TK, et al. IL-10 polymorphisms are associated with IL-10 production and clinical malaria in young children. Infect Immun. 2012;10:2316-22.

12. McGuire W, Knight JC, Hill AV, Allsopp CE, Greenwood BM, Kwiatkowski D. Severe malarial anemia and cerebral malaria are associated with different tumor necrosis factor promoter alleles. J Infect Dis. 1999;179:287-90.

13. Cabantous S, Poudiougou B, Traore A, Keita M, Cisse MB, Doumbo O, et al. Evidence that interferon-gamma plays a protective role during cerebral malaria. J Infect Dis. 2005;192:854-60.

14. Koch O, Awomoyi A, Usen S, Jallow M, Richardson A, Hull J, et al. IFNGR1 gene promoter polymorphisms and susceptibility to cerebral malaria. J Infect Dis. 2002;185:1684-7.

15. Aucan C, Walley AJ, Hennig BJW, Fitness J, Frodsham A, Zhang L, et al. Interferon-alpha receptor-1 (IFNAR1) variants are associated with protection against cerebral malaria in the Gambia. Genes Immun. 2003:4:275-82

16. Israelsson E, Maiga B, Kearsley S, Dolo A, Homann MV, Doumbo OK, et al. Cytokine gene haplotypes with a potential effect on susceptibility to malaria in sympatric ethnic groups in Mali. Infect Genet Evol. 2011;11:1608-15.

17. Mockenhaupt FP, Cramer JP, Hamann L, Stegemann MS, Eckert J, Oh $\mathrm{N}$ - $\mathrm{R}$, et al. Toll-like receptor ( $T L R)$ polymorphisms in African children: common TLR-4 variants predispose to severe malaria. Proc Natl Acad Sci USA. 2006;103:177-82.

18. Esposito S, Molteni CG, Zampiero A, Baggi E, Lavizzari A, Semino M, et al. Role of polymorphisms of toll-like receptor ( TLR ) containing adaptor protein ( TIRAP) and FCGR2A genes in malaria susceptibility and severity in Burundian children. Malar J. 2012;11:196.

19. Omar AH, Yasunami M, Yamazaki A, Shibata H, Ofori MF, Akanmori BD, et al. Toll-like receptor 9 (TLR9) polymorphism associated with symptomatic malaria: a cohort study. Malar J. 2012;11:168.

20. Greene JA, Sam-Agudu N, John CC, Opoka RO, Zimmerman PA, Kazura JW. Toll-like receptor polymorphisms and cerebral malaria: TLR2 $\triangle 22$ polymorphism is associated with protection from cerebral malaria in a case control study. Malar J. 2012;11:47.

21. Schröder NWJ, Schumann RR. Single nucleotide polymorphisms of Tolllike receptors and susceptibility to infectious disease. Lancet Infect Dis. 2005:5:156-64.

22. Parren PW, Warmerdam PA, Boeije LC, Arts J, Westerdaal NA, Vlug $A$, et al. On the interaction of IgG subclasses with the low affinity FcyRlla ( CD 32) on human monocytes, neutrophils, and platelets: analysis of a functional polymorphism to human IgG 2. J Clin Invest. 1992;90:1537-46

23. Israelsson E, Vafa M, Maiga B, Lysén A, Iriemenam NC, Dolo A, et al. Differences in Fcgamma receptor lla genotypes and IgG subclass pattern of anti-malarial antibodies between sympatric ethnic groups in Mali. Malar J. 2008;7:175

24. Omi K, Ohashi J, Patarapotikul J, Hananantachai H, Naka I, Looareesuwan $\mathrm{S}$, et al. Fcgamma receptor IIA and IIIB polymorphisms are associated with susceptibility to cerebral malaria. Parasitol Int. 2002;51:361-6.

25. Shi YP, Nahlen BL, Kariuki S, Urdahl KB, McElroy PD, Roberts JM, et al. Fcgamma receptor lla (CD32) polymorphism is associated with protection of infants against high-density Plasmodium falciparum infection. VII. Asembo Bay Cohort Project. J Infect Dis. 2001;184:107-11.

26. Ouma C, Keller CC, Opondo DA, Were T, Otieno RO, Otieno MF, et al. Association of FCgamma receptor IIA (CD32) polymorphism with malarial anemia and high-density parasitemia in infants and young children. Am J Trop Med Hyg. 2006;74:573-7.
27. Flori L, Delahaye NF, Iraqi FA, Fumoux F, Rihet P. TNF as a malaria candidate gene : polymorphism-screening and family-based association analysis of mild malaria attack and parasitemia in Burkina Faso. Genes Immun. 2005;6:472-80.

28. Cooke GS, Aucan C, Walley AJ, Segal S, Greenwood BM, Kwiatkowski DP, et al. Association of Fcy receptor lla (CD32) polymorphism with severe malaria in West Africa. Am J Trop Med Hyg. 2003;69:565-8.

29. Cherif MK, Sanou GS, Bougouma EC, Diarra A, Ouedraogo A, Dolo A, et al. Is FC gamma receptor IIA (FC gamma RIIA) polymorphism associated with clinical malaria and Plasmodium falciparum specific antibody levels in children from Burkina Faso? Acta Trop. 2015;142:41-6.

30. Natama HM, Vallbona ER, Somé MA, Zango SH, Sorgho H, Guetens P, et al. Malaria incidence and prevalence during the first year of life in Nanoro, Burkina Faso: a birth-cohort study. Malar J. 2018;17:163.

31. Branch $\mathrm{OH}$, Udhayakumar , Hightower AW, Oloo AJ, Hawley WA, Nahlen $\mathrm{BL}$, et al. A longitudinal investigation of $\lg \mathrm{G}$ and $\lg \mathrm{M}$ antibody responses to the merozoite surface protein-1 19-kiloDalton domain of Plasmodium falciparum in pregnant women and infants: associations with febrile illness, parasitemia, and anemia. Am J Trop Med Hyg. 1998;58:211-9.

32. Deloron P, Dubois B, Le Hesran JY, Riche D, Fievet N, Cornet M, et al. Isotypic analysis of maternally transmitted Plasmodium falciparum-specific antibodies in Cameroon, and relationship with risk of $P$. falciparum infection. Clin Exp Immunol. 1997;110:212-8.

33. Amaratunga C, Lopera-Mesa TM, Brittain NJ, Cholera R, Arie T, Fujioka $\mathrm{H}$, et al. A role for fetal hemoglobin and maternal immune lgG in infant resistance to Plasmodium falciparum malaria. PLoS ONE. 2011;6:e14798.

34. Kangoye DT, Nebie I, Yaro JB, Debe S, Traore S, Ouedraogo O, et al. Plasmodium falciparum malaria in children aged 0-2 Years: The role of foetal haemoglobin and maternal antibodies to two asexual malaria vaccine candidates (MSP3 and GLURP). PLOS ONE. 2014;9:e107965.

35. Simon AK, Hollander GA, McMichael A. Evolution of the immune system in humans from infancy to old age. Proc Soc Biol. 2014;2015(282):3085.

36. Natama HM, Moncunill G, Rovira-vallbona E, Sanz H, Sorgho H, Aguilar $\mathrm{R}$, et al. Modulation of innate immune responses at birth by prenatal malaria exposure and association with malaria risk during the first year of life. BMC Med. 2018;18:198.

37. Apinjoh T, Anchang-Kimbi J, Mugri R, Njua-Yafi C, Tata R, Chi H, et al. Determinants of infant susceptibility to malaria during the first year of life in South Western Cameroon. Open Forum Infect Dis. 2015;2:ofv012.

38. Kitua AY, Smith T, Alonso PL, Masanja H, Urassa H, Menendez C, et al. Plasmodium falciparum malaria in the first year of life in an area of intense and perennial transmission. Trop Med Int Health. 1996;1:475-84.

39. Hill AV. The immunogenetics of human infectious diseases. Annu Rev Immunol. 1998;16:593-617.

40. Zhao J, Ma L, Chen S, Xie Y, Xie L, Deng Y, et al. Association between Fc-gamma receptor lla (CD32) gene polymorphism and malaria susceptibility: a meta-analysis based on 6928 subjects. Infect Genet Evol. 2014;23:169-75.

41. Marquet S, Conte I, Poudiougou B, Argiro L, Cabantous S, Dessein H, et al. The IL17F and IL17RA genetic variants increase risk of cerebral malaria in two African populations. Infect Immun. 2016;84:590-7.

42. Hamann L, Bedu-Addo G, Eggelte TA, Schumann RR, Mockenhaupt FP. The toll-like receptor 1 variant S248N influences placental malaria. Infect Genet Evol. 2010;10:785-9.

43. Scott S, Mens PF, Tinto H, Nahum A, Ruizendaal E, Pagnoni F, et al. Community-based scheduled screening and treatment of malaria in pregnancy for improved maternal and infant health in The Gambia, Burkina Faso and Benin: study protocol for a randomized controlled trial. Trials. 2014;15:340.

44. Natama HM, Rovira-Vallbona E, Sorgho H, Somé MA, Traoré-Coulibaly $\mathrm{M}, \mathrm{Scott} \mathrm{S}$, et al. Additional screening and treatment of malaria during pregnancy provides further protection against malaria and nonmalarial fevers during the first year of life. J Infect Dis. 2018;217:1967-76.

45. WHO. Basic malaria microscopy: Part I Learner's Guide. 2nd ed. Geneva: World Health Organization; 2010.

46. Hofmann N, Mwingira F, Shekalaghe S, Robinson LJ, Mueller I, Felger I. Ultra-sensitive detection of Plasmodium falciparum by amplification of multi-copy subtelomeric targets. PLoS Med. 2015;12:e1001788.

47. Natama HM, Ouedraogo DF, Sorgho H, Rovira-Vallbona E, Serra-casas E, Somé MA, et al. Diagnosing congenital malaria in a high-transmission 
setting : clinical relevance and usefulness of P. falciparum HRP2-based testing. Sci Rep. 2017;7:2080.

48. Wittwer CT, Reed GH, Gundry CN, Vandersteen JG, Pryor RJ. High-resolution genotyping by amplicon melting analysis using LCGreen. Clin Chem. 2003;860:853-60.

49. R Core Team. R: a language and environment for statistical computing. Vienna: R Foundation for Statistical Computing; 2016.

50. Horita N, Kaneko T. Genetic model selection for a case-control study and a meta-analysis. Meta Gene. 2015;5:1-8.

51. Benjamini Y, Hochberg Y. Controlling the false discovery rate: a practical and powerful approach to multiple testing. J R Stat Soc. 1995;57:239-300.

52. Chang DM. Cellular signals for the induction of human interleukin-1 receptor antagonist. Clin Immunol Immunopathol. 1995;74:23-30.

53. Arend WP, Smith MF, Janson RW, Joslin FG. IL-1 receptor antagonist and IL-1 beta production in human monocytes are regulated differently. J Immunol. 1991;147:1530-6.

54. Maiga B, Dolo A, Touré O, Dara V, Tapily A, Campino S, et al. Fc gamma Receptor Ila-H131R polymorphism and malaria susceptibility in sympatric ethnic groups, Fulani and Dogon of Mali. Scand J Immunol. 2014;79:43-50.

55. Nasr A, Iriemenam NC, Troye-Blomberg M, Giha HA, Balogun HA, Osman OF, et al. Fc gamma receptor lla (CD32) polymorphism and antibody responses to asexual blood-stage antigens of Plasmodium falciparum malaria in Sudanese patients. Scand J Immunol. 2007;66:87-96.

56. Nasr A, Hamid O, Al-Ghamdi A, Allam G. Anti-malarial lgG subclasses pattern and FcyRlla (CD32) polymorphism among pregnancy-associated malaria in semi-immune Saudi women. Malar J. 2013:12:110.

57. Sinha S, Mishra SK, Sharma S, Patibandla PK, Mallick PK, Sharma SK, et al. Polymorphisms of TNF-enhancer and gene for FcyRlla correlate with the severity of falciparum malaria in the ethnically diverse Indian population. Malar J. 2008:7:13

58. Munde EO, Okeyo WA, Raballah E, Anyona SB, Were T, Ong'echa JM, et al. Association between Fcy receptor IIA, IIIA and IIIB genetic polymorphisms and susceptibility to severe malaria anemia in children in western Kenya. BMC Infect Dis. 2017;17:289.

59. Omi K, Ohashi J, Patarapotikul J, Hananantachai H, Naka I, Looareesuwan $\mathrm{S}$, et al. FCy receptor IIA and IIIB polymorphisms are associated with susceptibility to cerebral malaria. Parasitol Int. 2002;51:361-6.

60. Ouma C, Davenport GC, Garcia S, Kempaiah P, Chaudhary A, Were T, et al. Functional haplotypes of Fc gamma (Fcy) receptor (FcyRIIA and FcyRIIIB) predict risk to repeated episodes of severe malarial anemia and mortality in Kenyan children. Hum Genet. 2012;131:289-99.

61. Pleass RJ, Woof JM. Fc receptors and immunity to parasites. Trends Parasitol. 2001;17:545-51.

62. van de Winkel JG, Capel PJ. Human IgG Fc receptor heterogeneity: molecular aspects and clinical implications. Immunol Today. 1993:14:215-21.

63. Braga EM, Scopel KKG, Komatsu NT, da Silva-Nunes M, Ferreira MU. Polymorphism of the Fcgamma receptor IIA and malaria morbidity. J Mol Genet Med. 2005;1:5-10.

64. Warmerdam PA, van de Winkel JG, Vlug A, Westerdaal NA, Capel PJ. A single amino acid in the second Ig-like domain of the human FC gamma receptor II is critical for human IgG2 binding. J Immunol. 1991;147:1338-43.

65. John CC, Park GS, Sam-Agudu N, Opoka RO, Boivin MJ. Elevated serum levels of IL-1 ra in children with Plasmodium falciparum malaria are associated with increased severity of disease. Cytokine. 2008;41:204-8.

66. Jakobsen PH, McKay V, Morris-Jones SD, McGuire W, Van Hensbroek $M B$, Meisner $S$, et al. Increased concentrations of interleukin- 6 and interleukin-1 receptor antagonist and decreased concentrations of beta2-glycoprotein I in Gambian children with cerebral malaria. Infect Immun. 1994;62:4374-9

67. Armah HB, Wilson NO, Sarfo BY, Powell MD, Bond VC, Anderson W, et al. Cerebrospinal fluid and serum biomarkers of cerebral malaria mortality in Ghanaian children. Malar J. 2007;6:147.

68. Ataíde R, Mayor A, Rogerson SJ. Malaria, primigravidae, and antibodies: Knowledge gained and future perspectives. Trends Parasitol. 2014;30:85-94.

69. Mclntyre KW, Stepan GJ, Kolinsky KD, Benjamin WR, Plocinski JM, Kaffka $\mathrm{KL}$, et al. Inhibition of interleukin 1 (IL-1) binding and bioactivity in vitro and modulation of acute inflammation in vivo by IL-1 receptor antagonist and anti-IL-1 receptor monoclonal antibody. J Exp Med. 1991;173:931-9.
70. Gyan B, Goka B, Cvetkovic JT, Perlmann H, Lefvert AK, Akanmori B, et al. Polymorphisms in interleukin-1 $\beta$ and interleukin-1 receptor antagonist genes and malaria in Ghanaian children. Scand J Immunol. 2002;56:619-22.

71. Walley AJ, Aucan C, Kwiatkowski D, Hill AV. Interleukin-1 gene cluster polymorphisms and susceptibility to clinical malaria in a Gambian casecontrol study. Eur J Hum Genet. 2004;12:132-8.

72. Apinjoh TO, Anchang-Kimbi JK, Njua-Yafi C, Mugri RN, Ngwai AN, Rockett $\mathrm{KA}$, et al. Association of cytokine and toll-like receptor gene polymorphisms with severe malaria in three regions of cameroon. PLOS ONE. 2013;8:e81071.

73. Dinarello CA. Infection, fever, and exogenous and endogenous pyrogens: some concepts have changed. J Endotoxin Res. 2004;10:201-22.

74. Schofield L, Villaquiran J, Ferreira A, Schellekens H, Nussenzweig V. Gamma interferon, CD8+T cells and antibodies required for immunity to malaria sporozoites. Nature. 1987;330:664-6.

75. Vreden SGS, Van den Broek MF, Oettinger MC, Verhave JP, Meuwissen JHET, Sauerwein RW. Cytokines inhibit the development of liver schizonts of the malaria parasite Plasmodium berghei in vivo. Eur J Immunol. 1992:22:2271-5.

76. Prakash D, Fesel C, Jain R, Cazenave P-A, Mishra GC, Pied S. Clusters of cytokines determine malaria severity in Plasmodium falciparuminfected patients from endemic areas of Central India. J Infect Dis. 2006;194:198-207.

77. Vogetseder A, Ospelt C, Reindl M, Schober M, Schmutzhard E. Time course of coagulation parameters, cytokines and adhesion molecules in Plasmodium falciparum malaria. Trop Med Int Health. 2004;9:767-73.

78. John CC, Opika-Opoka R, Byarugaba J, Idro R, Boivin MJ. Low levels of RANTES are associated with mortality in children with cerebral malaria. J Infect Dis. 2006;194:837-45.

79. Pociot F, Molvig J, Wogensen L, Worsaae H, Nerup J. A Taql polymorphism in the human interleukin-1 $\beta$ (IL-1 $\beta)$ gene correlates with IL-1 $\beta$ secretion in vitro. Eur J Clin Invest. 1992;22:396-402.

80. Hernandez-Guerrero C, Monzon-Bordonaba F, Jimenez-Zamudio L, Ahued-Ahued R, Arechavaleta-Velasco F, Strauss JF, et al. In-vitro secretion of proinflammatory cytokines by human amniochorion carrying hyper-responsive gene polymorphisms of tumour necrosis factor- $a$ and interleukin-1 B. Mol Hum Reprod. 2003;9:625-9.

81. Ouma C, Davenport GC, Awandare GA, Keller CC, Were T, Otieno MF, et al. Polymorphic variability in the interleukin (IL)-1 beta promoter conditions susceptibility to severe malarial anemia and functional changes in IL1 beta production. J Infect Dis. 2008:198:1219-26.

82. Chen H, Wilkins LM, Aziz N, Cannings C, Wyllie DH, Bingle C, et al. Single nucleotide polymorphisms in the human interleukin-1B gene affect transcription according to haplotype context. Hum Mol Genet. 2006:15:519-29.

83. Santtila $\mathrm{S}$, Savinainen $\mathrm{K}$, Hurme M. Presence of the IL-1RA allele 2 (IL1RN*2) is associated with enhanced IL-1 beta production in vitro. Scand J Immunol. 1998;47:195-8.

84. Rogus J, Beck JD, Offenbacher S, Huttner K, lacoviello L, Latella MC, et al. IL1B gene promoter haplotype pairs predict clinical levels of interleukin1 beta and C-reactive protein. Hum Genet. 2008;123:387-98.

85. Couper KN, Blount DG, Riley EM. IL-10: the master regulator of immunity to infection. J Immunol. 2008;180:5771-7.

86. Wenisch C. Elevated serum levels of IL-10 and IFN- $\gamma$ in patients with acute Plasmodium falciparum malaria. Clin Immunol Immunopathol. 1995:74:115-7.

87. Hugosson E, Montgomery SM, Premji Z, Troye-Blomberg M, Björkman A. Higher IL-10 levels are associated with less effective clearance of Plasmodium falciparum parasites. Parasite Immunol. 2004;26:111-7.

88. Perera MK, Herath NP, Pathirana SL, Phone-Kyaw M, Alles HK, Mendis KN, et al. Association of high plasma TNF-alpha levels and TNF-alpha/IL-10 ratios with TNF2 allele in severe $P$. falciparum malaria patients in Sri Lanka. Pathog Glob Health. 2013;107:21-9.

89. Boeuf PS, Loizon S, Awandare GA, Tetteh JK, Addae MM, Adjei GO, et al. Insights into deregulated TNF and IL-10 production in malaria: Implications for understanding severe malarial anaemia. Malar J. 2012;11:253.

90. Jagannathan P, Eccles-James I, Bowen K, Nankya F, Auma A, Wamala $\mathrm{S}$, et al. IFNY/LL-10 co-producing cells dominate the CD4 response to malaria in highly exposed children. PLoS Pathog. 2014;10:e1003864. 
91. Peyron F, Burdin N, Ringwald P, Vuillez JP, Rousset F, Banchereau J. High levels of circulating IL-10 in human malaria. Clin Exp Immunol. 1994;95:300-3.

92. Manjurano A, Clark TG, Nadjm B, Mtove G, Wangai H, Sepulveda N, et al. Candidate human genetic polymorphisms and severe malaria in a Tanzanian population. PLOS ONE. 2012;7:e47463.

93. Domingues W, Kanunfre KA, Rodrigues JC, Teixeira LE, Yamamoto L, Okay TS. Preliminary report on the putative association of IL10 -3575 T/A genetic polymorphism with malaria symptoms. Rev Inst Med Trop Sao Paulo. 2016;58:1-10

94. Da Silva SS, Clark TG, Campino S, Suarez-Mutis MC, Rockett KA, Kwiatkowski DP, et al. Investigation of host candidate malaria-associated risk/protective SNPs in a Brazilian Amazonian population. PLoS ONE. 2012;7:e36692.

95. Legason ID, Pfeiffer RM, Udquim KI, Bergen AW, Gouveia MH, Kirimunda $\mathrm{S}$, et al. Evaluating the causal link between malaria infection and endemic Burkitt Lymphoma in Northern Uganda: a Mendelian randomization study. EbioMedicine. 2017;25:58-65.

\section{Publisher's Note}

Springer Nature remains neutral with regard to jurisdictional claims in published maps and institutional affiliations.
Ready to submit your research? Choose BMC and benefit from:

- fast, convenient online submission

- thorough peer review by experienced researchers in your field

- rapid publication on acceptance

- support for research data, including large and complex data types

- gold Open Access which fosters wider collaboration and increased citations

- maximum visibility for your research: over $100 \mathrm{M}$ website views per year

At BMC, research is always in progress.

Learn more biomedcentral.com/submissions 